\title{
Simulação numérica de um escoamento incompressível em uma cavidade quadrada utilizando o Método do Passo Fracionado e o Método da Penalidade
}

\author{
Numerical simulation of an incompressible flow in a square cavity using the Fractional Step \\ Method and the Penalty Method
}

\author{
Evanildo Franco de Jesus \\ Universidade Federal do Pampa (UNIPAMPA), Campus Bagé, RS, Brasil \\ evanildofj@yahoo.com.br \\ Francieli Aparecida Vaz \\ Universidade Federal do Pampa (UNIPAMPA), Campus Bagé, RS, Brasil \\ francieli.vaz@unipampa.edu.br
}

\section{Informações do Artigo}

(c) (i) 8 (8)

Histórico do Artigo

Submissão: 2 de março de 2018.

Aceite: 3 de maio de 2018.

\section{Palavras-chave}

Equações de Navier-Stokes Método das Diferenças Finitas

Método do Passo Fracionado

Método da Penalidade

Simulação Numérica

\section{Resumo}

A simulação numérica é amplamente utilizada na área de dinâmica dos fluidos, pois por meio do emprego de métodos numéricos pode-se analisar o comportamento de diferentes tipos de escoamentos de fluidos. Neste trabalho realizou-se a simulação numérica de um escoamento incompressível utilizando as equações de Navier-Stokes aplicadas para o escoamento de um fluido no interior de uma cavidade quadrada. Para a discretização dessas equações usou-se o método das Diferenças Finitas e para tratar o acoplamento da velocidade e pressão presentes nas equações de Navier-Stokes foram aplicados dois métodos: o método do Passo Fracionado e o método da Penalidade, facilitando assim a resolução dessas equações. Fazendo uso de um código escrito em linguagem $\mathrm{C}++$ foram realizadas simulações de escoamentos bidimensionais para 0 número de Reynolds 100, 400 e 1.000. Os resultados numéricos deste trabalho foram comparados com os resultados de referência disponíveis na literatura. O método do Passo Fracionado foi o método que gerou resultados mais satisfatórios quando comparado com o método da Penalidade, baseado nos resultados de referência.

\section{Abstract}

The numeric simulation is widely used in the fluid dynamics area, because through the use of numeric methods the behavior of different types of fluid flows can be analyzed. In this work the numeric simulation of an incompressible flow was accomplished using Navier-Stokes equations applied for the fluid flow inside square cavity. For discretization of these equations was used the Finite Difference method and to treat the coupling of the velocity and pressure present in the Navier-Stokes equations two methods were applied: the Fractional Step method and the Penalty method, thus the resolution of these equations is more easily obtained. Through a code written in $\mathrm{C}++$ language, two-dimensional flow simulations were performed for the Reynolds number 100, 400 and 1.000. The numerical results of this work were compared with the reference results available in the literature. The Fractional Step was the method that generated more satisfactory results when compared to the Penalty method, based on the reference results. 


\section{Introdução}

Inúmeros são os avanços dos estudos realizados na mecânica dos fluidos, ciência que se dedica a estudar os fluidos (substâncias que se encontram no estado líquido ou gasoso) em repouso ou em movimento. Os fluidos estão em todas as partes da natureza como o ar, a água, o sangue que circula nas veias e artérias no corpo humano (FORTUNA, 2000). Entender mais sobre esses escoamentos sempre foi um desafio para matemáticos, físicos e engenheiros, pois é fundamental para o aperfeiçoamento de seus projetos relacionados com escoamentos de fluidos. Várias equações foram criadas para tentar descrever o comportamento dos escoamentos de fluidos, entre essas as equações de Navier Stokes (ENS).

As equações de Navier-Stokes (ENS) são algumas das equações mais conhecidas e fundamentais na área da mecânica dos fluidos. Foram elaboradas por Louis Marie Henri Navier (17851836) engenheiro francês e pelo matemático inglês George Gabriel Stokes (1819-1903), entre suas finalidades ela é utilizada para descrever e analisar o comportamento de escoamentos de fluidos em vários meios. Desde sua criação as ENS proporcionaram um grande avanço tecnológico em áreas como engenharia mecânica, naval, automobilística, indústriais, entre outras, pois são utilizadas para desenvolver projetos que precisam estudar e melhorar a aerodinâmica de corpos para diminuir o atrito desses com o vento, como carros e aviões, em projetos de usinas hidrelétricas, nos estudos de fluxos sanguíneos, equipamentos médicos, estudo da dispersão de poluentes na atmosfera (SOUZA, 2013), enfim tudo que está relacionado e envolva fluxos de fluidos.

As ENS são classificadas como equações diferencias parciais (EDP) não lineares que possibilitam determinar campos de velocidade e pressão em problemas de escoamentos de fluidos (ÇENGEL; CIMBALA, 2007). São equações complexas e quando se trabalha com elas normalmente é realizado uma série de simplificações para diminuir o grau de dificuldade e facilitar os cálculos. Geralmente uma das primeiras medidas tomadas é considerar o fluido incompressível, tornando viável a obtenção de uma solução para o problema.

Os termos que compõe as ENS são formados por diferentes grandezas como, velocidade, pressão, densidade, viscosidade, entre outros que se não "tratados" podem causar mais tempo de execução em uma simulação numérica e outras complicações. Por exemplo, como forma de contornar esse problema, alguns pesquisadores como Buk Júnior (2007) e Souza (2013) em seus trabalhos costumam adimensionalizar essas equações; esse recurso proporciona como vantagem a economia de tempo e recursos computacionais. Quando esse recurso é adotado, pode-se tra- 
balhar com o escoamento laminar e turbulento usando números adimensionais, como o número de Reynolds.

Depois de realizar as devidas simplificações é necessário escolher um método numérico para discretizar as ENS. Dentre os vários métodos existentes, os mais usados são: o Método dos Elementos Finitos (MEF), Método dos Volumes Finitos (MVF) e o Método das Diferenças Finitas (MDF). Com o método numérico adequado é possível substituir as derivadas existentes nas equações diferenciais por expressões algébricas, facilitando a resolucão das ENS numericamente. É necessário também discretizar a região do problema (conhecida também como domínio) onde se quer obter a solução (FORTUNA, 2000), para isso escolhe-se alguns pontos no domínio e aplica-se para cada um desses as ENS discretizadas possibilitando a resolução computacional dessas equações.

Entre as técnicas de soluções criadas para resolver problemas sobre fluidos, a técnica numérica é a mais eficiente em relação ao tempo e ao custo, pois permite uma análise detalhada da região estudada. Atualmente é comum utilizar computadores para resolver problemas de escoamentos em que é difícil obter uma solução analítica ou experimental.

A área que utiliza computadores e métodos numéricos para resoluções desses problemas é conhecida como Dinâmica dos Fluidos Computacional (DFC). Constroem-se algoritmos para simular essas e outras equações para casos apresentando desde as geometrias mais simples até as mais complexas. Geralmente é necessário ter um conhecimento dos aspectos físicos do problema, para saber qual metodologia que se deva adotar (FORTUNA, 2000).

Com o objetivo de simular as equações de Navier-Stokes, testar e validar novos algoritmos numéricos, encontra-se disponível na literatura vários autores que utilizam o caso da cavidade quadrada com a intenção de analisar os resultados obtidos das simulações numéricas realizadas. O problema da cavidade quadrada com tampa móvel é considerado um clássico teste na solução numérica das ENS, permitindo realizar comparações qualitativas e quantitativas entre diversos métodos numéricos (PEREZ GUERREIRO et al., 1999).

Barros et al. (1999), como também Mendes (2007), utilizam o caso da cavidade quadrada para comparar e análisar métodos de desacoplamento entre pressão-velocidade presente nas equações de Navier-Stokes, empregando o método dos Volumes Finitos para discretização dessas. Para o mesmo caso Ghia et al. (1982) utilizam o método Multigrid para estudar o comportamento do escoamento para números altos de Reynolds. 
Neste trabalho realiza-se a simulação numérica das equações de Navier-Stokes considerando um escoamento incompressível, laminar e bidimensional no interior de uma cavidade quadrada com tampa móvel. O método das Diferenças Finitas é utilizado na discretização das ENS e para resolver o acoplamento da pressão e velocidade emprega-se dois métodos separadamente, método do Passo Fracionado e o método da Penalidade e posteriormente é realizada uma comparação entre esses. Os resultados numéricos deste trabalho são comparados com o trabalho de Ghia et al. (1982) que é amplamente utilizado para fins de comparação.

\section{Modelo Matemático}

A equação da continuidade 1 juntamente com as equações de Navier-Stokes 2 são utilizadas para a simulação do escoamento em uma cavidade quadrada. Por meio dessas é possível conhecer o campo de velocidade e/ou pressão.

Considerando um fluido newtoniano e incompressível estas equações na forma adimensionalizada apresentam a seguinte forma:

$$
\begin{gathered}
\vec{\nabla} \cdot \vec{V}=0 \\
\frac{\partial \vec{V}}{\partial t}+\vec{\nabla}(\vec{V} \vec{V})=-\vec{\nabla} P+\frac{1}{R e} \nabla^{2} \vec{V}
\end{gathered}
$$

onde $\vec{V}$ é a velocidade do escoamento, $P$ é a pressão e $R e$ é o número de Reynolds usado para determinar se o escoamento é laminar, transiente ou turbulento.

A Equação 1 garante a conservação da massa, ou seja, esta não apresenta alterações durante o escoamento. Pela Equação 2 tem-se uma relação estabelecida entre aceleração, pressão e forças que o fluido sofre durante o seu fluxo, permitindo assim determinar o campo de velocidade e/ou pressão.

Uma das dificuldades para resolver numericamente as equações de Navier-Stokes e da continuidade é a condição de incompressibilidade, cujo campo de velocidades deve ter divergência nula. Logo, a pressão passa a ser uma variável não relacionada a qualquer equação constitutiva; como consequência existe um acoplamento desconhecido entre pressão e velocidade que impede que as duas variáveis possam ser aproximadas independentemente (BUK JÚNIOR, 2007).

Para tratar da pressão e velocidade surgem diversos métodos; a seguir apresentam-se dois métodos utilizados neste trabalho, o método do Passo Fracionado e o método da Penalidade. Para a aproximação numérica das derivadas o método adotado é o das Diferenças Finitas. 


\subsection{Método do Passo Fracionado}

Considere a equação de Navier-Stokes adimensionalisada e discretizada no tempo:

$$
\frac{\vec{V}^{n+1}-\vec{V}^{n}}{\Delta t}=-\vec{\nabla} P^{n+1}-\vec{\nabla}(\vec{V} \vec{V})^{n}+\frac{1}{R e}\left(\nabla^{2} \vec{V}^{n}\right)
$$

Esse método consiste em dividir a Equação 3 em duas, conforme a seguir:

$$
\begin{gathered}
\frac{\vec{V}^{\star}-\vec{V}^{n}}{\Delta t}=-\vec{\nabla}(\vec{V} \vec{V})^{n}+\frac{1}{R e}\left(\nabla^{2} \vec{V}^{n}\right) \\
\frac{\vec{V}^{n+1}-\vec{V}^{\star}}{\Delta t}=-\vec{\nabla} P^{n+1}
\end{gathered}
$$

Ao efetuar esse processo surge uma variável $\left(\vec{V}^{\star}\right)$ denominada de variável intermediária.

Para obter a equação de Poisson 6, aplica-se o operador divergente $(\vec{\nabla} \cdot)$ na Equação 5 e considera-se o $\vec{\nabla} \cdot \vec{V}^{n+1}=0$.

$$
(\vec{\nabla} \cdot \vec{\nabla}) P^{n+1}=\frac{1}{\Delta t} \vec{\nabla} \cdot \vec{V}^{\star}
$$

A Equação 4 é denominada como equação de Burguer e é obtida quando desconsiderase o termo da pressão na ENS. A Equação 4 possibilita encontrar num primeiro passo um campo de velocidade intermediário que é utilizado para calcular-se o campo de pressão pela equação de Poisson 6. Ao final, tem-se o campo de velocidade do escoamento.

\subsection{Método da Penalidade}

Com o mesmo objetivo do método anterior, o método da Penalidade foi aplicado nas ENS. Esse método utiliza um mecanismo que possibilita o desacoplamento pressão e velocidade através de uma formulação que permite tratar um problema incompressível por meio de uma aproximação levemente compressível. Esse método é amplamente utilizado junto ao método de Elementos Finitos por pesquisadores como Buk Júnior (2007), Paskin (2016) e Souza (2013), porém nosso objetivo é usá-lo com o método das Diferenças Finitas.

A formulação penalizada é obtida estabelecendo uma relação entre a pressão e os campos de velocidades, tal que:

$$
P=-\lambda(\vec{\nabla} \cdot \vec{V})
$$


onde $\lambda$ é o parâmetro de penalização e pode ser expresso como $\lambda=c \mu$. Hughes et al. (1979) consideram o parâmetro $c$ uma constante que está relacionada com o tamanho de palavra do computador utilizado. Esse valor pode variar de acordo com cada caso estudado.

Para que a pressão permaneça finita o divergente do campo de velocidades deve tender a zero quando $\lambda$ tende ao infinito. Para isso o parâmetro $\lambda$ deve ser grande o necessário para que a compressibilidade introduzida seja mínima (PASKIN, 2016).

Substituindo a Equação 7 na Equação 2 obtém-se:

$$
\frac{\partial \vec{V}}{\partial t}=-\vec{\nabla}(\vec{V} \vec{V})+\frac{c}{R e} \vec{\nabla}(\vec{\nabla} \cdot \vec{V})+\frac{1}{R e} \nabla^{2} \vec{V}
$$

Após efetuado todo esse processo, obtém-se uma equação somente em relação aos campos de velocidades.

\section{Estudo Numérico da Cavidade Quadrada}

A cavidade quadrada foi escolhida por apresentar uma geometria simples em que é fácil definir as condições de contorno, implementar e testar algoritmos numéricos, analisar os campos de pressão e velocidades, como também as características do escoamento em seu interior.

Na Figura 1 estão definidas a geometria da cavidade quadrada e as condições de contorno. As partes laterais e inferior são paredes fixas; na parte superior tem-se uma tampa móvel e o interior da cavidade está ocupado com um fluido. As condições de contorno utilizadas são as de Dirichlet, que definem a solução da variável na fronteira (ZILL; CULLEN, 2001). Como mencionado anteriormente, a simulação será para o caso bidimensional considerando $\vec{V}=(u, v)$.

Inicialmente o fluido está em repouso no interior da cavidade, saindo dessa condição quando a tampa movél recebe uma velocidade máxima e constante $u=1$. No momento em que a tampa é deslocada, seu movimento acaba puxando o fluido adjacente à ela, devido às tensões viscosas presentes, o que acaba originando o escoamento do fluido (FORTUNA, 2000).

Para simular o escoamento na cavidade quadrada utilizou-se o método das Diferenças Finitas juntamente com o método do Passo Fracionado e o método da Penalidade. O método das Diferenças Finitas foi aplicado para discretizar as equações de Navier-Stokes, no qual foi possível substituir as derivadas presentes nessas equações por aproximações de diferenças finitas. Para os termos 
transientes presentes nas ENS usou-se aproximações progressivas e para os termos convectivos e difusivos, aproximações centrais.

Para os casos simulados considerou-se o número de Reynolds igual 100, 400 e 1.000, e uma malha $81 \times 81$ quadrangular e uniforme. Os resultados numéricos das velocidades $u$ e $v$ são comparados com os resultados numéricos encontrados no trabalho de Ghia et al. (1982), que também simulam o caso da cavidade quadrada para números de Reynolds variando de 100 até 1.000, utilizando uma malha uniforme $129 \times 129$.

O algoritmo utilizado para simulação numérica foi escrito e desenvolvido em linguagem C++ no ambiente de desenvolvimento integrado e livre $\mathrm{Dev} \mathrm{C}_{+}+$e para fazer a análise gráfica dos resultados utilizou-se o Scilab versão 5.5.2.

Figura 1 - Geometria e condições de contorno da cavidade quadrada.

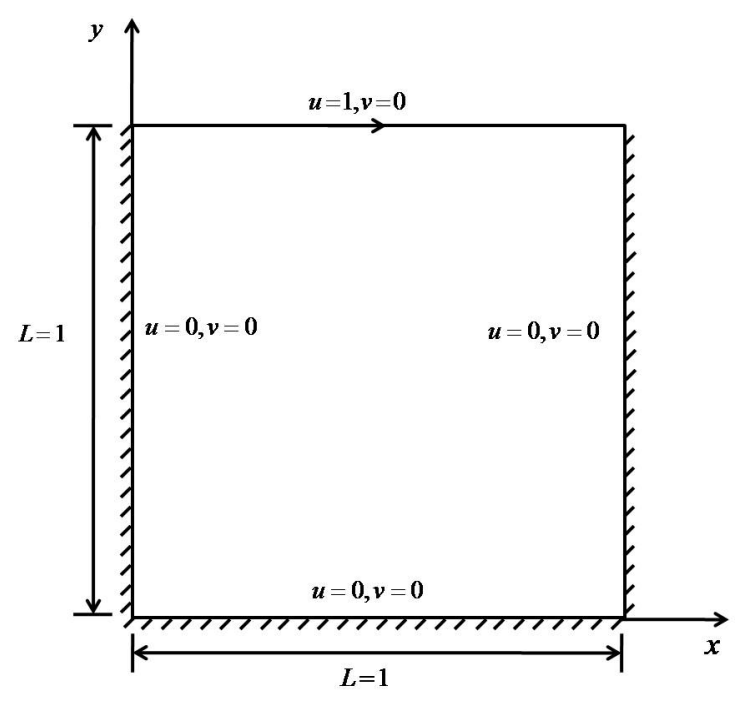

Fonte: Adaptado de Barros et al. (1999, p. 6).

\subsection{Resultados Numéricos}

Foram analisados os perfis das velocidades $u$ e $v$ em relação ao eixo central da cavidade nas posições horizontal e vertical. Nas Figura 2 e Figura 3 são apresentados os perfis de velocidade $u$ em $x=0.5 L$ e $v$ em $y=0.5 L$ para Reynolds 100. Observando os gráficos nota-se que os resultados numéricos com o método do Passo Fracionado se aproximaram mais dos resultados da referência de Ghia et al. (1982) quando comparado com o método da Penalidade. 
Figura 2 - Perfil da velocidade $u$ para $R e=100$.

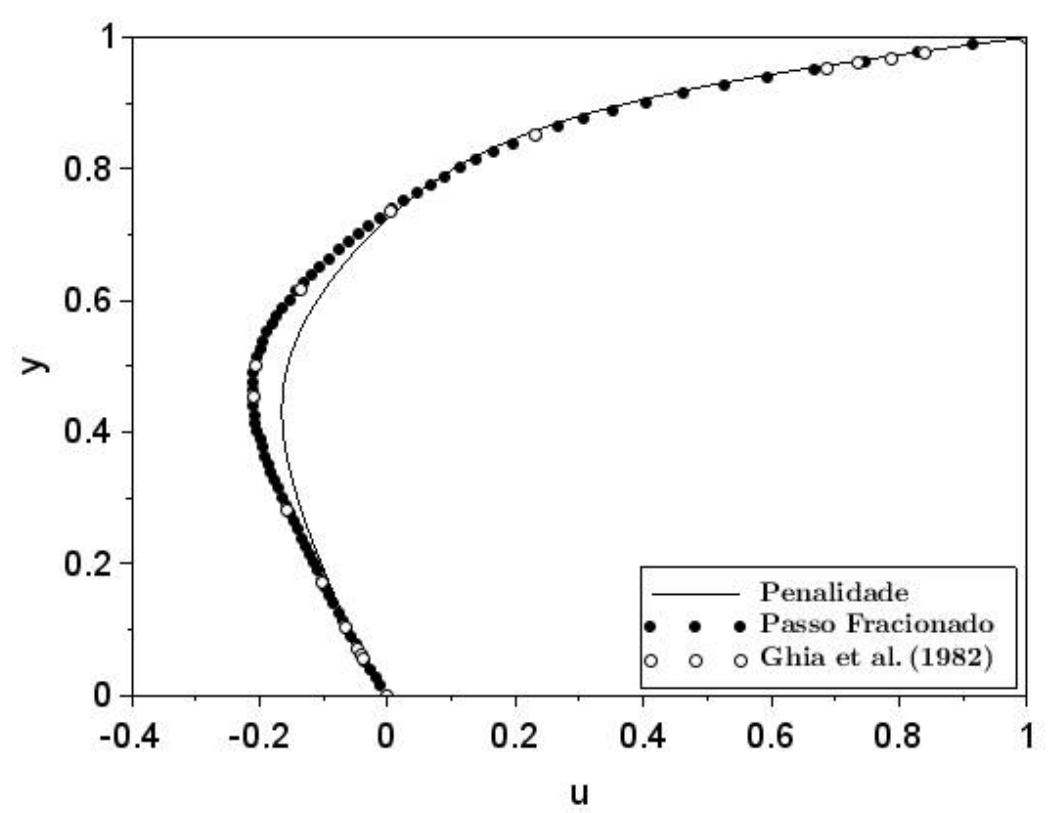

Fonte: Elaboração dos autores.

Figura 3 - Perfil da velocidade $v$ para $R e=100$.

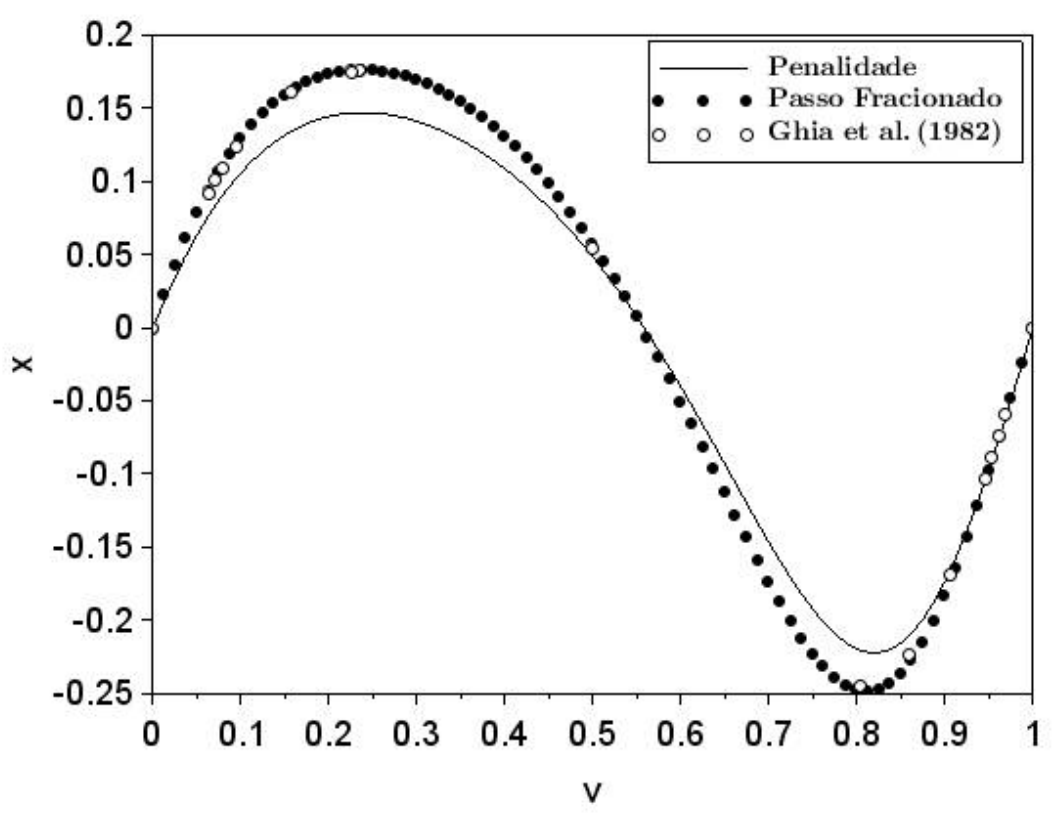

Fonte: Elaboração dos autores. 
Nas Figura 4 e Figura 5 os perfis de $u$ em $x=0.5 L$ e $v$ em $y=0.5 L$ foram avaliados para Reynolds 400. O método do Passo Fracionado apresentou resultados em concordância com os da referência. Nota-se que à medida que o número de Reynolds aumenta, o método da Penalidade começa a apresentar aproximações afastadas da referência. Um dos motivos para este comportamento está relacionado ao tamanho da malha; se essa for refinada, a diferença entre os resultados dos métodos diminuem, como será analisado mais adiante.

Para valores baixos de Reynolds como 100 e 400 os termos difusivos e advectivos presentes nas equações de Navier-Stokes não são tão significativos; esses começam a ser expressivos para valores maiores, como Reynolds 1.000, influenciando nas características do escoamento do fluido no interior da cavidade, pois os campos de velocidades e pressão sofrem variações relevantes.

As Figura 6 e Figura 7 mostram os perfis das velocidade $u$ e $v$ em relação aos eixos horizontal e vertical da cavidade para Reynolds 1.000. O método do Passo Fracionado ainda continua apresentando boa concordância com os da referência, ao contrário do método da Penalidade que se afasta dos resultados de referência; mas cabe ressaltar que a malha utilizada nesse trabalho é menor que a malha utilizada pela referência. Também é necessário destacar que para Reynolds 1.000 o escoamento já apresenta velocidades maiores que os casos anteriores. Para esse número a pressão provoca grandes alterações nas características do escoamento, o que faz com que o método da Penalidade apresente um comportamento instável na medida em que aumenta o número de Reynolds.

A fim de analisar e avaliar o comportamento do método da Penalidade para uma malha mais refinada, foi simulado o caso da cavidade para Reynolds 100, 400 e 1.000 com uma malha $201 \times 201$. Nas Figura 8, Figura 9 e Figura 10, apresenta-se apenas o perfil da velocidade $u$ em $x=0.5 \mathrm{~L}$ com a finalidade de comparar o método da Penalidade para diferentes malhas. Podese observar que quando se refina a malha obtêm-se resultados mais precisos. Esse resultado sugere que, para obter resultados melhores a malha deveria ser mais refinada, porém limitações computacionais impediram testes nesse sentido.

Para todas as simulações foi adotado $\Delta t=10^{-5}$ e o critério de parada considera a variação relativa de uma passo para o outro menor que $10^{-8}$. Apesar das características geométricas da cavidade serem simples, o escoamento do fluido em seu interior apresentou movimentos recirculatórios que podem ser considerados complexos à medida em que aumentou-se o número de Reynolds. 
Figura 4 - Perfil da velocidade $u$ para $R e=400$.

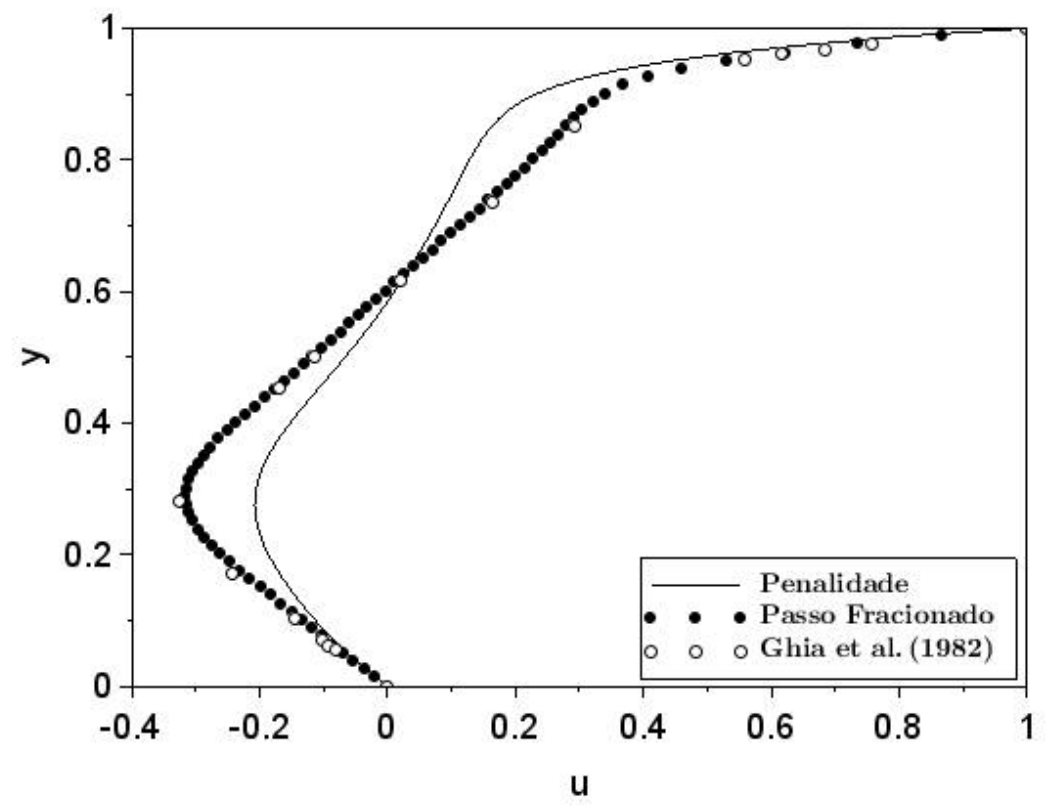

Fonte: Elaboração dos autores.

Figura 5 - Perfil da velocidade $v$ para $R e=400$.

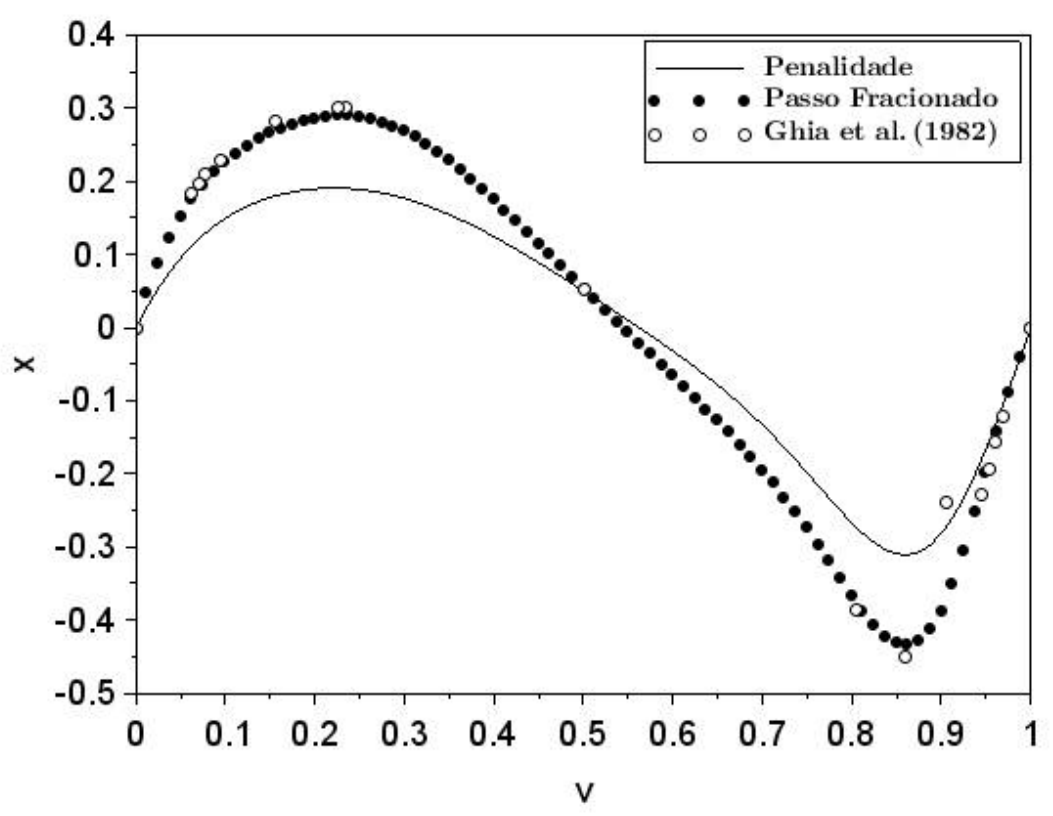

Fonte: Elaboração dos autores. 
Figura 6 - Perfil da velocidade $u$ para $R e=1.000$.

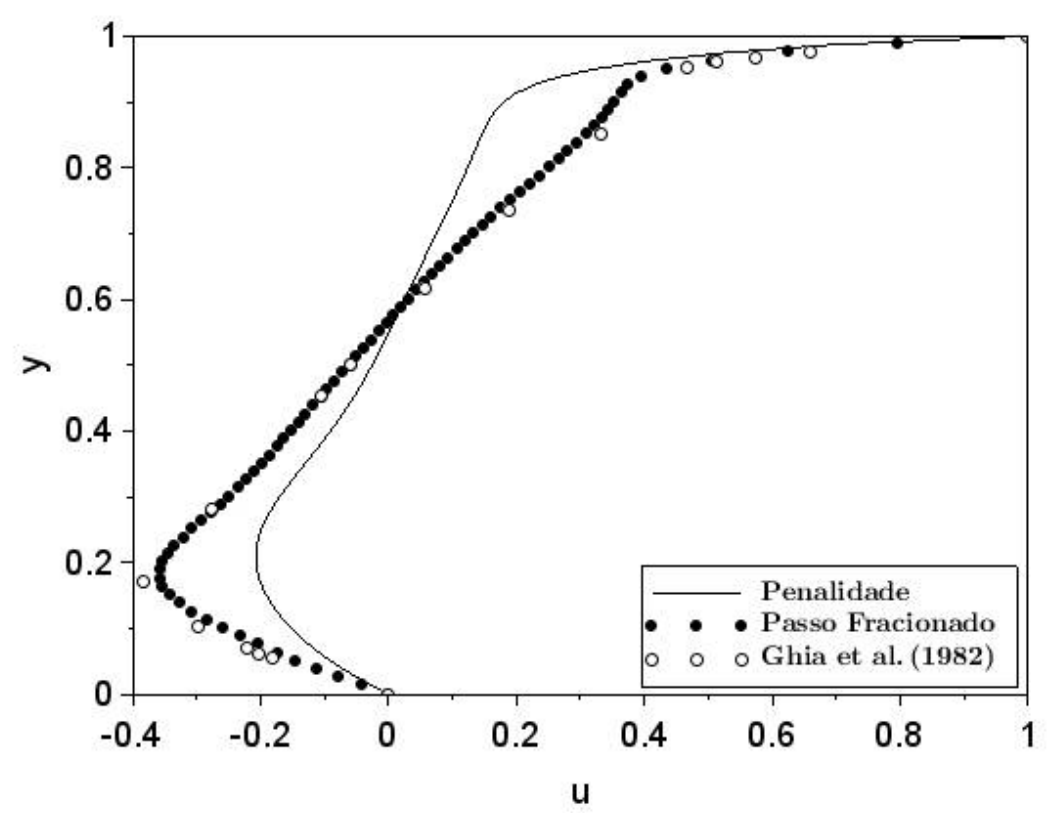

Fonte: Elaboração dos autores.

Figura 7 - Perfil da velocidade $v$ para $R e=1.000$.

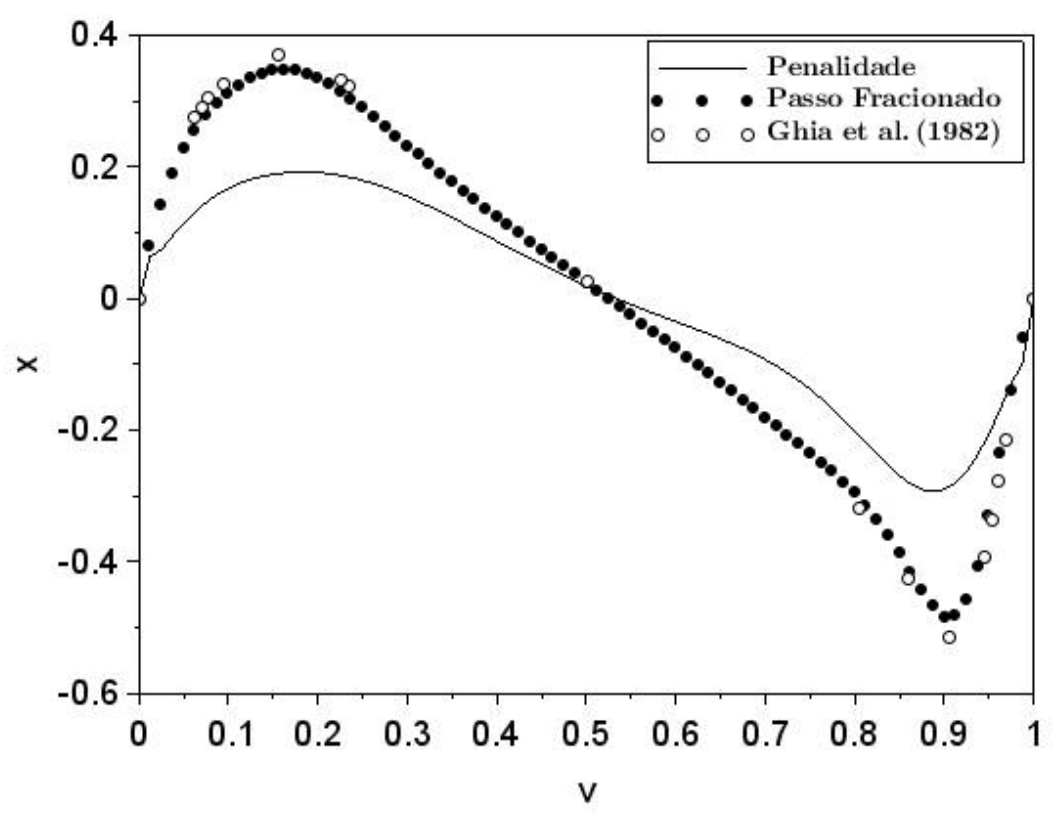

Fonte: Elaboração dos autores. 
Figura 8 - Perfil da velocidade $u$ para Reynolds 100 com diferentes malhas para o método da Penalidade.

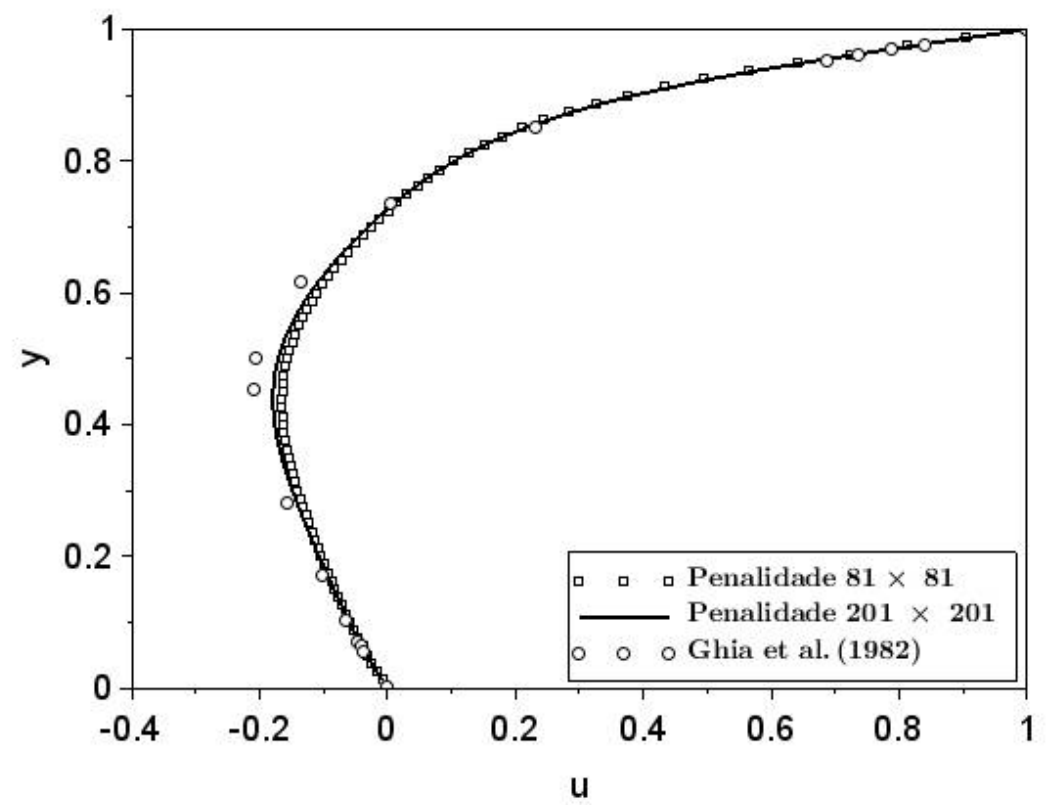

Fonte: Elaboração dos autores.

Figura 9 - Perfil da velocidade $u$ para Reynolds 400 com diferentes malhas para o método da Penalidade.

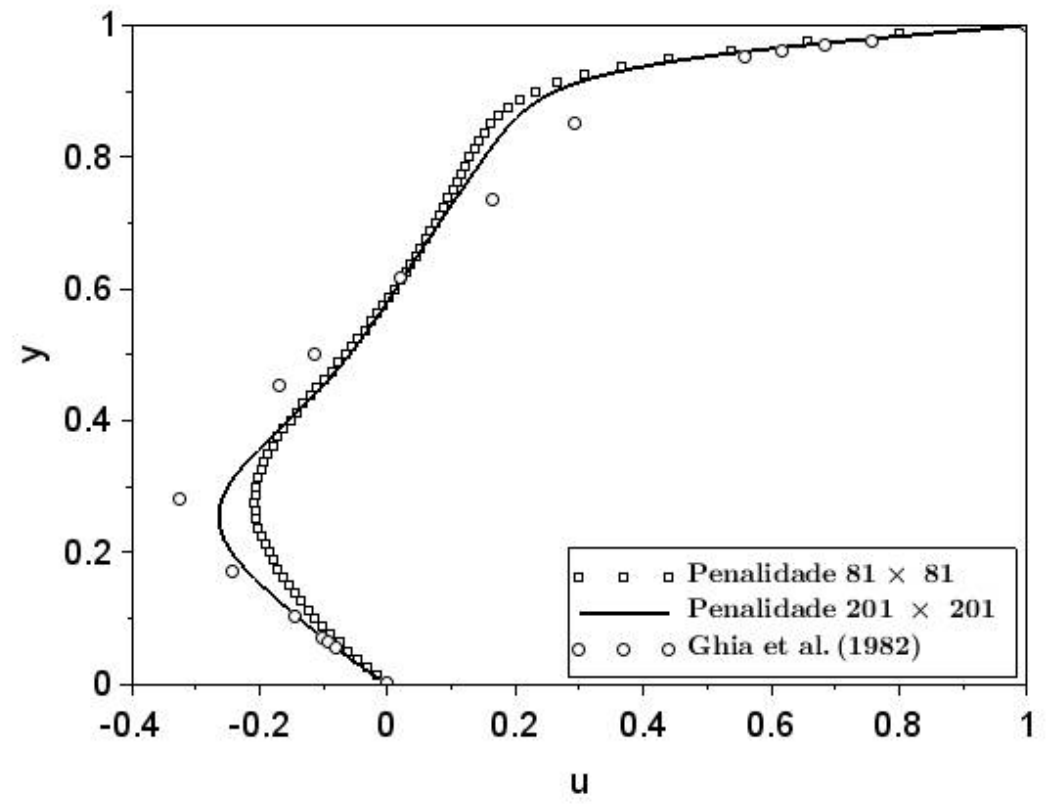

Fonte: Elaboração dos autores. 
Figura 10 - Perfil da velocidade $u$ para Reynolds 1.000 com diferentes malhas para o método da Penalidade.

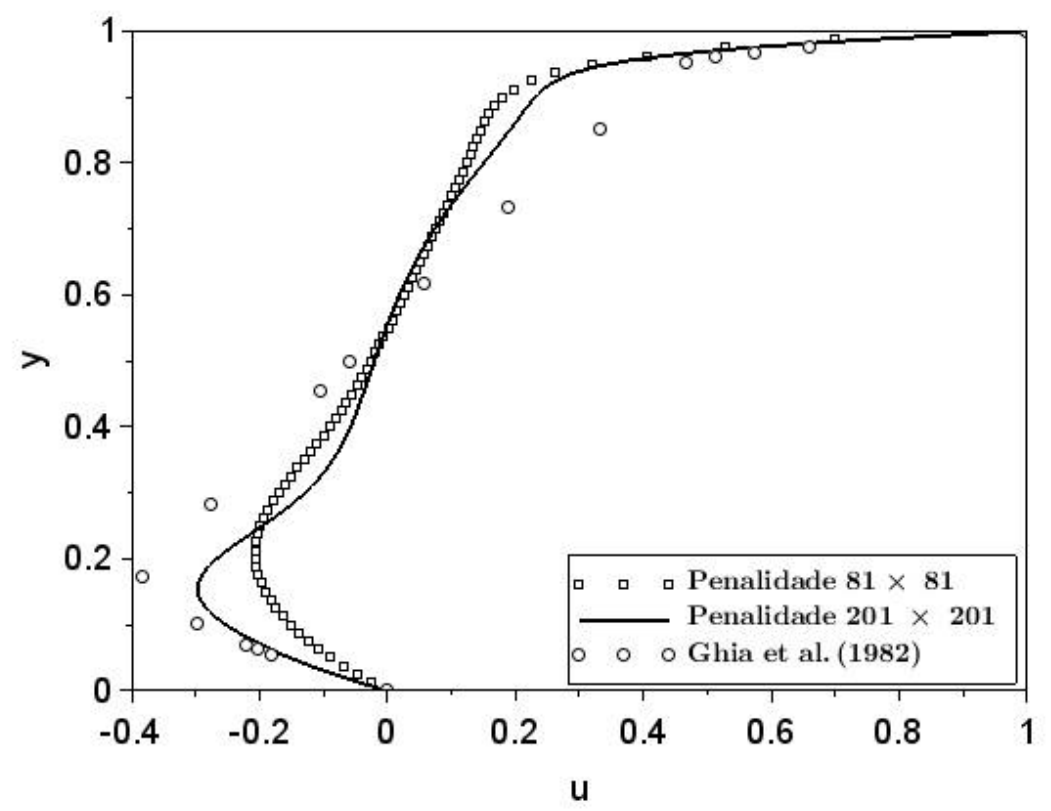

Fonte: Elaboração dos autores.

Nas Tabela 1 e Tabela 2 são apresentados os valores da raiz do quadrado médio do erro, também denominado desvio padrão dos resíduos, calculados a partir dos resultados numéricos obtidos pelo método do Passo Fracionado e o método da Penalidade comparados com os dados de referência para as velocidades $u$ e $v$ nas posições centrais ao eixo da cavidade.

Assim como já mencionado nos comentários das figuras anteriores, os valores das Tabela 1 e Tabela 2 confirmam que a solução numérica obtida através do método do Passo Fracionado é a que se aproxima mais dos resultados de referência. Outra observação é a comparação entre diferentes valores de Reynolds, o modelo que mais se aproxima do ideal é com Reynolds 100, com o aumento do número de Reynolds ambos os métodos começam a ficar mais instáveis, devido ao aumento da velocidade e os efeitos de recirculações presentes no escoamento.

Tabela 1 - Análise do desvio padrão dos resíduos para velocidade $u$ no eixo central da cavidade.

\begin{tabular}{ccc}
\hline Reynolds & Passo Fracionado & Penalidade \\
\hline 100 & 0,02229 & 0,02940 \\
400 & 0,03174 & 0,15335 \\
1000 & 0,04205 & 0,10441 \\
\hline \multicolumn{3}{c}{ Fonte: Elaboração dos autores. }
\end{tabular}


Tabela 2 - Análise do desvio padrão dos resíduos para velocidade $v$ no eixo central da cavidade.

\begin{tabular}{ccc}
\hline Reynolds & Passo Fracionado & Penalidade \\
\hline 100 & 0,01134 & 0,01863 \\
400 & 0,01516 & 0,07938 \\
1000 & 0,02792 & 0,13930 \\
\hline \multicolumn{3}{c}{ Fonte: Elaboração dos autores. }
\end{tabular}

\section{Conclusão}

A elaboração e comparação de métodos de desacoplamento da pressão e velocidade em relação as equações de Navier-Stokes é muito comum, principalmente pelo fato dessas equações serem essenciais para diversos estudos em inúmeras áreas. O método do Passo Fracionado já é um método muito conhecido e utilizado em diversos trabalhos devido sua eficiência e precisão nos resultados gerados, já o método da Penalidade não é tão conhecido e utilizado devido a sua formulação ser um pouco complexa, o que acaba influenciando nos resultados. Esse método tem alguns parâmetros com valores que ainda não estão precisamente bem definidos e que provocam instabilidade nos resultados como foram constatados neste trabalho.

Os resultados numéricos obtidos para o caso da cavidade quadrada com o método do Passo Fracionado foram satisfatórios quando comparado com os resultados numéricos da referência utilizada, diferente do método da Penalidade que se mostrou menos eficaz. Para malhas mais refinadas o método da Penalidade gerou soluções melhores que se aproximaram das soluções numéricas obtidas com a do método do Passo Fracionado, mas ainda assim a diferença dos resultados entre eles é significativa. Espera-se que com novos testes e estudos consiga-se aperfeiçoar e ajustar os parâmetros desse método e assim obter resultados melhores.

\section{Referências}

BARROS, A. M.; MARTINS, S. B. C.; RENGEL, J. E.; SPHAIER, S. H. Estudo comparativo de diferentes métodos de desacoplamento pressão-velocidade nas equações de Navier-Stokes. In: CONGRESSO BRASILEIRO DE ENGENHARIA MECÂNICA, 15., 1999, São Paulo. Anais ..., São Paulo: ABCM, 1999. Dísponivel em:

<http://abcm.org.br/anais/cobem/1999/portugues/AACJFB.htm>. Acesso em: 07 jun. 2017.

BUK JÚNIOR, L. Estudo numérico do escoamento ao redor de um cilindro fixo. 2007. 62 f. Dissertação (Mestrado em Engenharia Mecânica) - Escola Politécnica da Universidade de São Paulo, São Paulo, 2007. 
ÇENGEL, Y. A; CIMBALA, J. M. Mecânica dos fluidos: fundamentos e aplicações. São Paulo: McGraw-Hill, 2007.

FORTUNA, A. O. Técnicas computacionais para dinâmica dos fluidos: conceitos básicos e aplicações. São Paulo: Edusp, 2000.

GHIA, U.; GHIA, K. N.; SHIN, C. T. High-Re solutions for incompressible flow using the Navier-Stokes equations and multigrid method. Journal of Computational Physycs, Ohio, v. 48, p. 387-620, 1982.

HUGHES, T. J. R.; LIU, W. K.; BROOKS, A. Finite elements analysis of incompressible viscous flows by the penalty function formulation. Journal of Computational Physycs, Califórnia, v. 30, p. 1-60, 1979.

MENDES, R. Análise do acoplamento pressão-velocidade nas equações de Navier-Stokes utilizando o método dos volumes finitos baseado em elementos e solução acoplada. 2007. 102 f. Dissertação (Mestrado em Engenharia Mecânica) - Universidade Federal de Santa Catarina, Florianópolis, 2007.

PASKIN, L. Solução computacional das equações de Navier-Stokes em uma formulação penalizada de elementos finitos. 2016. $51 \mathrm{f}$. Trabalho de Conclusão de Curso (Bacharelado em Engenharia Naval e Oceânica) - Escola Politécnica da Universidade Federal do Rio de Janeiro, Rio de Janeiro, 2016.

PEREZ GUERREIRO, J. S.; RAMOS, R.; PIMENTEL, L. C. G. Analytical solution of the lid driven cavity problem for Reynolds number tending towards zero. In: CONGRESSO BRASILEIRO DE ENGENHARIA MECÂNICA, 15., 1999, São Paulo. Anais ..., São Paulo: ABCM, 1999. Dísponivel em: <http://abcm.org.br/anais/cobem/1999/portugues/AAAIJI.htm>. Acesso em: 04 jan. 2018.

SOUZA, M. M. Solução das equações de Navier-Stokes para fluidos incompressíveis via elementos finitos. 2013. 113 f. Dissertação (Mestrado em Matemática Aplicada) - Universidade Federal do Rio Grande do Sul, Porto Alegre, 2013.

ZILL, D. G.; CULLEN, M. R. Equações diferenciais. v. 1, 3. ed. São Paulo: Pearson Makron Books, 2001. 